\title{
Nanomechanical behaviour of open-cell nanoporous metals: homogeneous versus thickness-dependent porosity
}

\author{
D. Esqué-de los Ojos ${ }^{1 *}$, J. Zhang $^{1}$, J. Fornell ${ }^{1}$, E. Pellicer ${ }^{1}$, J. Sort $^{2}$ \\ ${ }^{1}$ Departament de Física, Universitat Autònoma de Barcelona, E-08193 Bellaterra, Spain \\ ${ }^{2}$ Institució Catalana de Recerca i Estudis Avançats (ICREA) and Departament de Física, \\ Universitat Autònoma de Barcelona, E-08193 Bellaterra, Spain
}

\begin{abstract}
Two different nanoporous materials, porous copper prepared by dealloying and porous nickel prepared by electrodeposition, have been studied by means of nanoindentation experiments at different maximum applied loads. While nanoporous $\mathrm{Cu}$ is homogeneous along its cross-section, the electrodeposited Ni films show a graded porosity, with smaller pores and thicker pore walls close to the film's surface. The mechanical properties of the two materials have been extracted using a methodology based on scaling laws and subsequent interpretation has been performed using finite element simulations. Two different deformation mechanisms are observed for nanoporous $\mathrm{Cu}$ and nanoporous Ni, respectively. Dealloyed porous copper behaves as an homogeneous material without evident effect of densification and with mechanical properties that are independent of the applied load. Given this homogeneity, it is possible to fit the entire loading - unloading curve for different maximum applied loads with a single set of mechanical properties. Conversely, electrodeposited porous nickel shows a decrease in the reduced Young's modulus, an increase in yield stress and a constant hardness when the maximum applied load during nanoindentation is increased. While the decrease in the reduced Young's modulus can be explained in the context of thickness inhomogeneity of the electrodeposited porous nickel (i.e., increase of porosity with depth), this cannot explain, and actually would go against, the observed increase in the yield stress, which is instead associated to the decrease in the ligament size.
\end{abstract}

\section{Submitted to Mechanics of Materials \\ December 3, 2015}

Keywords: Dealloying; Electrodeposition; Finite element analysis (FEA)

Mechancial properties; Nanoindentation; Porous materials; Size effects

\footnotetext{
*Corresponding author. e-mail: danielesque@gmail.com
} 


\section{Introduction}

Owing to their extraordinarily high surface area, nanoporous materials have become ideal candidates for a widespread range of technological applications, both in chemistry (e.g., gas sensors, catalysis, batteries, gas molecules separators) (Liu et al. 2011; Otsubo et al., 2012; Sun et al., 2005; Xiong et al., 2012), as well as in optical, electronic and magnetic devices (Huan et al., 2011; Pellicer et al., 2013). From a structural point of view, porous materials are being used for impact-energy absorption and in lightweight construction, as well as in various types of thermal insulation and acoustic damping (Linul et al., 2013).

Several strategies are currently being pursued for the preparation of nanoporous films. These include evaporation-induced self-assembly, dealloying, or electrodeposition (Brezesinski et al., 2006; Malgras et al., 2015; Scaglione et al., 2012) among others. Electrodeposition (either using hard templates - e.g. anodic aluminum oxide - or soft templates - e.g. block copolymers in the electrolytic bath) and de-alloying are particularly suited for the preparation of metallic porous alloys (sometimes referred to as metallic foams). Selective de-alloying techniques typically produce materials with an open sponge-like structure of interconnecting ligaments and pore sizes of a few nm. During de-alloying, the least noble component in a solid solution is leached from the alloy, while the noblest elements undergo surface diffusion and agglomeration, forming the sponge-like metallic structure (Malgras et al., 2015).

Irrespectively of the application, the mechanical properties of porous materials are of uppermost importance to ensure robustness and endurance of any device in which they are integrated. In fact, despite their extended utilization in industry, porous materials suffer from the drawback of being usually rather brittle. Therefore, the study of their mechanical properties by uniaxial tension, macroscopic compression or torsion experiments is often not possible. For this reason, nanoindentation has become one of the most appropriate methods to measure the mechanical properties of these materials since this technique keeps the induced deformation constrained into very local length scales. However, proper interpretation of the mechanical properties of porous materials using nanoindentation remains still rather challenging. First, a densified zone can form underneath the indenter during indentation, thus precluding a clear-cut assessment of the properties of the initially non-deformed material. Secondly, most of the models existing in the literature to correlate mechanical properties (i.e., hardness and Young's modulus) with the porosity degree 
are only applicable to some particular arrangements of porous networks (e.g., honeycomb-like geometries or close-cell pore structures) (Pellicer et at., 2012; Ramakrishnan and Arunachalam, 1993). Remarkably, while the porosity degree is often taken into account in these models, the important role played by the pore size itself on the resulting mechanical performance is often overlooked. Furthermore, most mechanical studies so far have only focussed on fully homogeneous porous films or foams. The mechanical response of heterogeneous (i.e., thickness-dependent porosity) nanoporous alloys has essentially not been investigated.

In this work, the mechanical response during nanoindentation of homogenous nanoporous $\mathrm{Cu}$ films, prepared by dealloying, and that of heterogeneous nanoporous Ni (i.e., with graded porosity across thickness) directly grown by electrodeposition from a block polymer-containing solution, are compared. Interpretation of the experimental results is supported with finite element simulations. While the properties of homogeneous nanoporous $\mathrm{Cu}$ are found to be rather independent of the applied indentation load, the Young's modulus of porosity-graded nanoporous Ni decreases for higher loads, while the opposite trend is observed in the corresponding values of yield stress. Such results are ascribed to the structural evolution of nanoporous Ni with depth, i.e., to the interplay between the increase of the average pore size (and porosity degree) and the decrease of the ligament size as the distance from the film's surface increases.

\section{Materials and Methods}

\subsection{Copper-Zinc dealloying}

$\mathrm{Cu}_{20} \mathrm{Zn}_{80} 2 \mathrm{~mm}$-thick sheets were purchased from Goodfellow. Nanoporous copper $(\mathrm{Cu})$ samples were obtained by dealloying these sheets in $85 \mathrm{wt} \% \mathrm{H}_{3} \mathrm{PO}_{4}$ for 4 days following the methodology described by Chung Cheng and Hodge, 2012. Scanning Electron Microscopy (SEM) equipped with Energy-dispersive X-ray (EDX) was used to prove the Zn depletion. The morphology of the obtained nanoporous layer was observed by SEM. Further verification of Zn depletion was carried out by X-ray diffraction (XRD).

Prior to indentation and SEM imaging, the sample was hot embedded in a conductive resin and polished with diamond suspension to mirror like appearance. Figure 1 corresponds to a cross-section SEM image of the dealloyed copper where an homogeneous microstructure across its depth can be observed. 


\section{$2.2 \quad$ Nickel electrodeposition}

Nanoporous nickel (Ni) film was obtained by potentiostatic electrodeposition in a single compartment double-jacketed three-electrode cell. Si|Ti $(25 \mathrm{~nm}) \mid \mathrm{Au}(125 \mathrm{~nm})$ substrate was used as cathode with working area of $0.25 \mathrm{~cm}^{2}$ exposed to the electrolyte. A platinum spiral served as counter electrode, which was positioned vertically facing the working electrode. A double junction $\mathrm{Ag} \mid \mathrm{AgCl} 3 \mathrm{M} \mathrm{KCl}$ electrode $(\mathrm{E}=+0.210 \mathrm{~V}$ versus standard hydrogen electrode) was utilized as reference electrode. Electrodeposition was conducted using a PGSTAT302N Autolab potentiostat/galvanostat (Ecochemie). Electrolyte was prepared by mixing the pluronic P123 amphiphilic block copolymer surfactant and aqueous solution of $0.4 \mathrm{M} \mathrm{NiSO}_{4}, 0.5 \mathrm{M} \mathrm{NaSO}_{4}, 0.2$ $\mathrm{M}$ boric acid and $0.5 \mathrm{~g} / \mathrm{L}$ saccharine. To prepare the mixture, $4 \mathrm{~g}$ of $\mathrm{P} 123$ was heated to $60^{\circ} \mathrm{C}$ in a beaker prior to the addition of the aqueous phase and mixed manually for 5-10 min at constant heat. Then certain amount of ethanol was added to the mixture to assure a ratio of $0.2 \mathrm{~g}$ P123 per $\mathrm{mL}$ of ethanol. The resulting solution was subjected to vigorous magnetic stirring until it showed a turbid milky appearance. The nanoporous Ni was obtained at room temperature by applying a constant potential of $-1.8 \mathrm{~V}$ for $1200 \mathrm{~s}$.

The concentration of surfactant was set at $8 \mathrm{wt} \%$ which is above the critical micelle concentration of P123. The hydrophilic Au substrate serves as aggregation site for P123, as the surfactant gathers spontaneously at the solid-liquid interface. The surfactant micelle directs the $\mathrm{Ni}^{2+}$ ions towards the substrate and acts as structure-directing agent. As depicted in Figure 2 (a), the growth of Ni follows a quasi-perfect layer-by-layer process, in which the pore size decreases gradually along the vertical direction. This layer-by-layer growth fashion is evident from the corresponding SEM picture, see Figure 2 (b). Layer III, which corresponds to the first stages of electrodeposition, exhibits the largest pore size. As deposition proceeds, a second Ni layer is formed on top of the first porous Ni. This second layer (II) features smaller pore size. Consequently, the uttermost layer (layer I) has the smallest pore size (around 10-20 nm). Contrary to the average pore size, the width of the pore walls (i.e., lateral size of the ligament) progressively increases with film thickness, from 10 - $15 \mathrm{~nm}$ (layer III) to 50 - $60 \mathrm{mn}$ (layer I). 


\subsection{Nanoindentation experiments, extraction of mechanical properties and finite element simulations}

Nanoindentation experiments were performed in load-control mode, using a UMIS instrument from Fischer-Cripps Laboratories equipped with a Berkovich pyramidal-shaped diamond tip. The maximum applied load ranged from 2 to $25 \mathrm{mN}$. The thermal drift was always kept below $0.05 \mathrm{~nm} / \mathrm{s}$. At least 10 indents were performed at each maximum applied load to verify the accuracy of the indentation data. The same protocol was followed for both porous $\mathrm{Cu}$ and $\mathrm{Ni}$.

For the extraction of mechanical properties, the hardness and the reduced Young's modulus values were evaluated at the beginning of the unloading segment by means of the method of Oliver and Pharr, 1992. In order to estimate the yield stress, $\sigma_{\mathrm{ys}}$, we used the methodology described in Alcalá et al., 2009; Casals and Alcalá, 2005. It is well known that during sharp indentation of a homogeneous material, the relation between the applied load and the displacement of the indenter can be expressed through (Cheng and Cheng, 1999; Giannakopoulos et al., 1994; Lawn and Howes, 1981):

$$
P=K h_{\mathrm{s}}^{2},
$$

being $P$ the applied load, $h_{\mathrm{s}}$ the indenter's displacement and $K$ a constant that depends on the indented material. The unloading stage can be fitted using (Oliver and Pharr, 1992):

$$
P_{\mathrm{u}}=B\left(h_{\mathrm{s}}-h_{\mathrm{r}}\right)^{\mathrm{m}},
$$

where $B$ and $m$ are fitting parameters and $h_{\mathrm{r}}$ is the remaining $h_{\mathrm{s}}$ at $P=0$. Additionally, the Sneddon's relation states that:

$$
E_{\text {eff }}=\left.\frac{\eta}{\sqrt{A_{\max }}} \frac{\mathrm{d} P}{\mathrm{~d} h}\right|_{h_{\max }},
$$

being $E_{\text {eff }}$ the effective Young's modulus, $\eta$ is assumed to vary with the indenter's shape (Cao et al., 2007; Meza et al., 2008; Xu and Li, 2008) and it is equal to 0.793 for a Berkovich indenter (Alcalá et al., 2009), and $A_{\max }$ is the projected contact area between the material and the indenter at $\left(h_{\max }, P_{\max }\right) . E_{\text {eff }}$ can be written as: 


$$
E_{\text {eff }}=\left(\frac{1}{E_{\mathrm{r}}}+\frac{1-\nu_{\mathrm{i}}^{2}}{E_{\mathrm{i}}}\right)^{-1},
$$

where $\nu_{\mathrm{i}}$ and $E_{1}$ are the Poisson's coefficient and Young's modulus of the indenter, respectively, and $E_{\mathrm{r}}$ the reduced Young's modulus of the material under indentation. The slope at $\left(h_{\max }\right.$, $\left.P_{\max }\right)$ during unloading can be calculated through:

$$
\left.\frac{\mathrm{d} P}{\mathrm{~d} h}\right|_{h_{\max }}=\frac{P_{\max }}{h_{\max }-h_{\mathrm{e}}}=\frac{P_{\max }}{h_{\max }\left(1-h_{\mathrm{e}} / h_{\max }\right)},
$$

where $h_{\mathrm{e}}$ is the penetration depth at which the tangent curve at $\left(h_{\max }, P_{\max }\right)$ crosses the $h_{\mathrm{s}}$ axis. Figure 3 shows an schematic of a loading - unloading indentation curve including the expressions for the loading and unloading stages and the expression for the slope of the unloading stage.

Once parameters $B, m$ and $h_{\mathrm{r}}$ in Eq. 2 are fitted, $\mathrm{dP} /$ dh evaluated at $h_{\max }$ can be obtained by differentiating Eq. 2 and $h_{\mathrm{e}}$ can be calculated through Eq. (5). Combining Eqs. (1), (3) and (5) for $\left(h_{\max }, P_{\max }\right)$ one can write:

$$
E_{\text {eff }}=\eta \sqrt{K \bar{p}}\left(1-\frac{h_{\mathrm{e}}}{h_{\mathrm{max}}}\right)^{-1},
$$

being $\bar{p}=P / A$ the obtained hardness from nanoindentation experiments and $A$ the projected contact area between the material and the indenter.

Finally, once having the values for $E_{\text {eff }}$ and $\bar{p}$, one can use the expression (Casals and Alcalá, 2005):

$$
\frac{\bar{p}}{\sigma_{0.1}}=A_{1}+A_{2} \cdot \exp \left(\frac{-E_{\mathrm{eff}} / \sigma_{0.1}}{A_{3}}\right)+A_{4} \cdot \exp \left(\frac{-E_{\mathrm{eff}} / \sigma_{0.1}}{A_{5}}\right)
$$

to obtain $\sigma_{0.1}$, a characteristic stress corresponding to an uniaxial deformation of 0.1. Expressions for $A_{1}, A_{2}, A_{3}, A_{4}$ and $A_{5}$ can be found in Table 7 for Eq. (A.1) in Casals and Alcalá, 2005. Relation between $\sigma_{0.1}$ and $\sigma_{\text {ys }}$ can be written as:

$$
\sigma_{\mathrm{ys}}^{n-1}=\frac{(0.1 E)^{n}}{\sigma_{0.1}}
$$

where $E$ is the Young's modulus and $n$ is the strain hardening coefficient during an uniaxial test.

With the extracted mechanical properties, we performed finite element (FE) simulations with the commercial ABAQUS finite element code under a full integration scheme. As the porous structure was not considered during simulations, the finite element mesh, constructed following 
a refining strategy, comprised two-dimensional 43,359 four-node axisymmetric quadrilateral elements and the Berkovich indenter was modelled using an equivalent conical indenter with an azimuthal angle equal to $65.3^{\circ}$. Simulations were performed under frictionless contact conditions and large-strain formulations. A linear elastic-perfectly plastic relation was assumed in the simulations with the Von Mises flow theory of plasticity, allowing to correlate directly $\sigma_{0.1}$ to $\sigma_{\mathrm{ys}}$, as the strain hardening coefficient $n$ was taken to be equal to zero.

\section{Results and discussion}

Figure 4 shows the nanoindentation curves at different maximum applied loads (Fig. 4 (a)) and the extracted mechanical properties (Figs. $4(\mathrm{~b})-(\mathrm{d})$ ) for the dealloyed porous $\mathrm{Cu}$. From Fig. 4 (a) it can be seen that the applied load $(P)$ - penetration depth $\left(h_{\mathrm{s}}\right)$ curves at different maximum applied loads $P_{\max }$ are coherent with each other, i.e., the loading stages match rather well, except for $P_{\max }=15 \mathrm{mN}$. Figs. 4 (b) and (c) show, respectively, the extracted values for the reduced Young's modulus $\left(E_{\mathrm{r}}\right)$ and hardness $\bar{p}$, according to Oliver and Pharr, 1992, as a function of the maximum penetration depth $h_{\max }$ for the different $P_{\max }$. Given the scattering, one can observe that the values for $E_{\mathrm{r}}$ and $\bar{p}$ do not show a significant tendency to increase, as one could expect on the event of a densification process. Analogously, Fig. 4 (d) shows the evolution of the scaled hardness $\left(\bar{p} / \sigma_{0.1}\right)$ with $h_{\max }$ for different applied $P_{\max }$, being the value of $\bar{p} / \sigma_{0.1}$ practically constant. For a homogeneous bulk metal one can expect that the constraint factor $C=\bar{p} / \sigma_{0.1}=2.6$ (Casals and Alcalá, 2005; Mata et al., 2002). However, by introducing porosity, this value will be necessarily reduced due to reduction of hydrostatic pressure (DomingoRoca et al., 2015; Zeeshan et al., 2015). Fig. 4 (a) also includes FE simulations capturing the experimental $P-h_{\mathrm{s}}$ curves. Interestingly, a single set of mechanical properties was used for the simulations, namely $E_{\mathrm{r}}=13.0 \mathrm{GPa}$ and $\sigma_{0.1}=\sigma_{\mathrm{ys}}=478.9 \mathrm{MPa}$, supporting the idea that the present dealloyed porous $\mathrm{Cu}$ is homogeneous across the entire thickness. These values were obtained by averaging the extracted mechanical properties in Table 1 for $P_{\max }$ from 2.5 to $15 \mathrm{mN}$. The resulting simulated $P-h_{\mathrm{s}}$ curve was able to capture the experiments for the entire range of applied loads fairly well (here, just simulations for $P_{\max }=10 \mathrm{mN}$ and $15 \mathrm{mN}$ are included for representation purposes). Most likely, simulations were not able to perfectly capture the loading stages due to slight structural changes in the dealloyed porous $\mathrm{Cu}$ during indentation. Table 1 
summarizes the extracted mechanical properties according to what has been explained in Section 2.3 and also the relative density of the porous $\mathrm{Cu}, \rho^{*} / \rho_{\mathrm{s}}$, being $\rho^{*}$ and $\rho_{\mathrm{s}}$ the densities of the porous and bulk materials respectively, calculated using Eq. 9 (Gibson and Ashby, 1997):

$$
E_{\mathrm{r}}=C_{2} E_{\mathrm{s}}\left(\frac{\rho^{*}}{\rho_{\mathrm{s}}}\right)^{n},
$$

where $E_{\mathrm{r}}$ is the reduced Young's modulus of the porous material. As the morphology of the dealloyed porous $\mathrm{Cu}$ approaches to an open-cell foam, the values $C_{2}=1$ and $n=2$ can be assumed (Gibson and Ashby, 1997; Williford et al., 2005) giving as a result $\rho^{*} / \rho_{\mathrm{s}}=0.33$ (taking $E_{\mathrm{s}}=120 \mathrm{GPa}$ as the reduced Young's modulus of bulk $\mathrm{Cu}$ ). This relative density is not far from the value of 0.20 that one could expect from the starting material $\left(\mathrm{Cu}_{20} \mathrm{Zn}_{80}\right)$, if $\mathrm{Zn}$ was fully removed during the dealloying. The slight discrepancy could be due to: (i) small amounts of $\mathrm{Zn}$ still present in the dealloyed $\mathrm{Cu}$ (although not easily detected by EDX) or (ii) $C_{2}$ and $n$ not being exactly equal to 1 and 2 , respectively. It is worth mentioning that to extract $\sigma_{0.1}$, as explained in Section 2.3, the used methodology was first thought for bulk-homogeneous materials. However, we have proven that the dealloyed porous $\mathrm{Cu}$ can be understood as a homogeneous material, therefore this methodology can apply. A proof of that is the excellent agreement between experiments and simulations (using a single set of parameters) and also between the values of Young's moduli obtained through the method of Oliver and Pharr and the presented methodology, as it can be seen in Table 1.

In the same fashion as for $\mathrm{Cu}$, Figure 5 shows the $P-h_{\mathrm{s}}$ curves (Fig. 5 (a)) and extracted mechanical properties (Figs. $5(\mathrm{~b})-(\mathrm{d})$ ) for different $P_{\max }$ for electrodeposited Ni. Contrary to what was observed in Fig. 4 (a) for $\mathrm{Cu}$, here the loading stages for different $P_{\max }$ do not overlap so much with each other, anticipating heterogeneity across the electrodeposited porous Ni sample. Furthermore, Fig. 5 (b) shows that $E_{\mathrm{r}}$ decreases with $h_{\max }$ for different applied $P_{\max }$, being this coherent with what was shown in Fig. 2 (a) and (b), i.e., a material whose porosity increases with depth. Hence, if porosity increases it is natural to obtain a reduction in $E_{\mathrm{r}}$, not considering, though, densification effects that, on the contrary, would increase $E_{\mathrm{r}}$. However, this explanation seems to fail in Figs. 5 (c) and (d) that show, respectively, the variation of $\bar{p}$ and $\bar{p} / \sigma_{0.1}$ with $h_{\max }$. From Fig. 5 (c) one can see that hardness remains approximately constant for the whole range of $h_{\max }$. Instead, the constraint factor, $\bar{p} / \sigma_{0.1}$, decreases with $h_{\max }$ similar, to $E_{\mathrm{r}}$. Then, since $\bar{p} / \sigma_{0.1}$ decreases while $\bar{p}$ remains constant with $h_{\max }$, it is clear that the 
yield stress $\sigma_{\mathrm{ys}}$ increases with $P_{\max }$. Here, as for the dealloyed $\mathrm{Cu}$, one can consider that $\sigma_{0.1}$ $\sim \sigma_{\mathrm{ys}}$, as it corresponds to a linear elastic - perfectly plastic material with no strain hardening, being this idea supported by Schwaiger et al., 2003. The decrease of $E_{\mathrm{r}}$ and $\bar{p} / \sigma_{0.1}$ with $h_{\max }$ is rather straightforward to understand taking into account that the present porous electrodeposited Ni shows an increased porosity with depth. As $h_{\max }$ increases, the material experiences less hydrostatic pressure, resulting in a progressively lower constraint factor. However, this does not explain the increase of $\sigma_{\mathrm{ys}}$ with $P_{\max }$. Actually, compaction of the material would certainly induce an increase of $\sigma_{\mathrm{ys}}$ but it would also increase $E_{\mathrm{r}}$. Nonetheless, the works by Biener et al., 2005 and Hodge et al., 2007 provided an answer for this apparent contradiction. These authors studied the yield strength of nanoporous open-cell Au foams for different relative densities $\left(\rho^{*} / \rho_{\mathrm{S}}\right)$. Although $\rho^{*}$ was decreasing, indicating an increment of porosity, the yield strength increased, as in our study. The explanation for this phenomenon was that the ligaments' size was reduced as $\rho^{*}$ became lower, leading to an effect analogous to the Hall-Petch relationship, where the characteristic length scale was, instead of the grain diameter, the ligament size:

$$
\sigma^{*}=C_{\mathrm{s}}\left[\sigma_{\mathrm{o}}+k \cdot L^{-1 / 2}\right] \cdot\left(\frac{\rho^{*}}{\rho_{\mathrm{s}}}\right)^{3 / 2},
$$

where $\sigma^{*}$ is the yield strength of the porous metal, $C_{\mathrm{s}}$ is a fitting parameter, $\sigma_{\mathrm{o}}$ is the yield strength of the bulk material, $k$ is the Hall-Petch type coefficient for the theoretical yield strength of the metal, $L$ is the ligament size and $\rho^{*}$ and $\rho_{\mathrm{s}}$ the densities of the porous and bulk materials respectively. Eq. 10 is the result of combining a Hall-Petch type of relation:

$$
\sigma_{\mathrm{yc}}=\sigma_{\mathrm{o}}+k \cdot L^{-1 / 2}
$$

(being $\sigma_{\mathrm{yc}}$ the yield strength of a ligament with size $L$ ) with the Gibson and Ashby, 1997 scaling prediction for foams:

$$
\sigma^{*}=C_{2} \sigma_{\mathrm{s}}\left(\frac{\rho^{*}}{\rho_{\mathrm{s}}}\right)^{3 / 2},
$$

with coefficient $C_{2}=0.3$ according to Gibson and Ashby, 1997 and $\sigma_{\mathrm{s}} \equiv \sigma_{\mathrm{o}}$ is the yield strength of the bulk material.

For the present nanoporous $\mathrm{Ni}$, given its morphology, it was possible to estimate the ligament size at the top surface of the electrodeposited film (i.e., $L \sim 50-60 \mathrm{~nm}$ ) and the first layer (III) 
, i.e., $L \sim 10-15 \mathrm{~nm}$. Table 2 summarizes the extracted mechanical properties according to Section 2.3 along with the different relative densities using Eq. 9 for open-cell porosity and $E_{\mathrm{s}}=$ $200 \mathrm{GPa}$. Taking $\sigma_{\mathrm{ys}}=\sigma^{*}=4.0 \mathrm{GPa}$, the bulk value for Ni $\sigma_{\mathrm{s}}=21.8 \mathrm{MPa}$ (Carlton and Ferrari, 2007; Thompson, 1975), and $k_{\mathrm{Ni}}=7 \mathrm{GPa} \sqrt{n m}$ (El-Sherik et al., 1992; Hughes et al., 1986) then, from Eq. 10 one can obtain $C_{\mathrm{s}}=3.2-4.0$. Having the values of $\sigma_{\mathrm{ys}}=\sigma^{*}$ in Table 2 for different $P_{\max }$ and the corresponding values of $\rho^{*} / \rho_{\mathrm{s}}$ along with the fitting value $C_{\mathrm{s}}$, now one can calculate the corresponding ligament sizes that would produce the different values of $\sigma_{\mathrm{ys}}$. These values are listed in Table 2 and they are in rather good agreement with the SEM observations (Fig. 2). As for the case of the porous dealloyed $\mathrm{Cu}$, the extracted values for the Young's moduli with the method of Oliver and Pharr, 1992 and the present methodology are in excellent agreement. Also, this methodology was previously used in the framework of $\mathrm{Cu}$ single crystalline thin films on top of silica ( $\mathrm{Si}$ ) substrates (where the thin film/substrate interface acts as an inhomogeneity), with excellent results (Esqué-de los Ojos et al., 2014).

As in Fig. 4 (a) for the dealloyed porous $\mathrm{Cu}$, here we also performed $\mathrm{FE}$ simulations of the indentation experiments on electrodeposited porous Ni, Fig. 5(a). Given the evolution on the mechanical properties shown in Fig. 5(b) for the reduced Young's modulus and for the extracted yield stress in Table 2, in this case it was not possible to use a single set of parameters for the simulations, but different values of $E_{\mathrm{r}}$ and $\sigma_{\mathrm{ys}}$ were used for each $P_{\max }$, in accordance with Table 2 .

\section{Conclusions}

Here we have studied the mechanical behaviour of porous $\mathrm{Cu}$ obtained by dealloying and porous $\mathrm{Ni}$ prepared by electrodeposition, combining nanoindentation experiments with finite element simulations. The following are the most remarkable conclusions reached during the present study.

- For dealloyed porous $\mathrm{Cu}$ it has been shown that, as a result of its homogeneous structure, mechanical properties such as the reduced Young's modulus, hardness and yield stress do not vary significantly with different maximum applied loads. This enables to capture the applied load - penetration depth experimental indentation curves with a single set of mechanical properties through finite element simulations. As no evolution of the mechanical properties was observed, densification effects could be essentially disregarded up to $15 \mathrm{mN}$ 
maximum applied load.

- For electrodeposited porous Ni, the reduced Young's modulus and constraint factor decrease with the maximum applied load, while hardness stays constant. This variation of the mechanical properties precludes the use of a unique set of mechanical properties to fit the entire applied load - displacement indentation curves through finite element simulations. Actually, with each set of mechanical properties it is, at most, only possible to capture through finite element simulations the corresponding maximum load and the unloading stage, but not the loading stage. This is due to the inherent structure heterogeneity of the sample.

- Concerning the evolution in the mechanical properties of electrodeposited porous $\mathrm{Ni}$, the reduction of the Young's modulus and constraint factor with $P_{\max }$ can be understood from the increase of the porosity with depth, but this is in principle contradictory with the constancy of hardness and the increase of yield stress. To overcome this paradox, the critical role played by the ligament size was taken into account. The very small ligament sizes act similar to the small crystallite sizes in the Hall-Petch relation, in the sense that as the ligament size is reduced the yield stress increases. SEM images indeed reveal that while the porosity is increased, the ligament size is reduced across the electrodeposited porous $\mathrm{Ni}$ sample.

Acknowledgments- J. Z. is grateful to the China Scholarship Council (CSC) for the PhD grant. E.P. acknowledges the Spanish Ministerio de Economía y Competitividad (MINECO) for the Ramon y Cajal contract (RYC-2012-10839). Partial financial support from the 2014-SGR-1015 project from D.G.U. Catalunya and the SPIN-PORICS Consolidator Grant - 2014 from the European Research Council are also acknowledged.

\section{References}

Alcalá J., Esqué-de los Ojos D., Rodríguez S.A., 2009. The role of crystalline anisotropy in mechanical property extractions through Berkovich indentationJ. Mat. Res. 24, 1235 - 1244.

Biener J., Hodge A.M., Hamza A.V., Hsiung L.M., Satcher J.H, Jr., 2005. Nanoporous Au: A high yield strength material. J. Appl. Phys. 97, 024301. 
Brezesinski T., Groenewolt M., Gibaud A., Pinna N., Antonietti M., Smarsly B.M., 2006. Evaporation-induced self-assembly (EISA) at its limit: Ultrathin, crystalline patterns by templating of micellar monolayers. Adv. Funct. Mater. 18, 2260 - 2263.

Cao Y.P., Dao M., Lu J., 2007. A precise correcting method for the study of the superhard material using nanoindentation tests J. Mater. Res. 22, 1255 - 1264.

Carlton C.E., Ferreira P.J., 2007. What is behind the inverse HallPetch effect in nanocrystalline materials? Acta Mater. 55, 3749 - 3756.

Casals O., Alcalá J., 2005. The duality in mechanical property extractions from Vickers and Berkovich instrumented indentation experiments. Acta Mater. 53, 3545 - 3561.

Cheng Y.-T., Cheng C.-M., 1999. Scaling relationships in conical indentation of elastic perfectly plastic solids Int. J. Solids and Struct. 36, $1231-1243$.

Chung Cheng I., Hodge A.M., 2012. Morphology, oxidation, and mechanical behavior of nanoporous $\mathrm{Cu}$ foams. Adv. Eng. Mater. 14, $219-226$.

Domingo-Roca R., Esqué-de los Ojos D., Guerrero M., Pellicer E., Baró M.D., Suriñach S., Sort J., 2015. Nanomechanical behaviour of 3D porous metal-ceramic nanocomposites $\mathrm{Bi} / \mathrm{Bi}_{2} \mathrm{O}_{3}$ films. Mater. Sci. Eng. A 626, $150-158$.

El-Sherik A.M., Erb U., Palumbo G., Aust, K.T., 1992. Deviations from hall-petch behaviour in as-prepared nanocrystalline nickel. Scripta Metall. et Mater. 27, 1185 - 1188.

Esqué-de los Ojos D., Očenášek J., Alcalá J., 2014. Sharp indentation crystal plasticity finite element simulations: Assessment of crystallographic anisotropy effects on the mechanical response of thin fcc single crystalline films. Comp. Mater. Sci. 86, 186 - 192.

Giannakopoulos A.E., Larsson P.L., Vestergaard R., 1994. Analysis of Vickers indentation. Int. J. Solids and Struct. 31, $2679-2708$.

Gibson L.J., Ashby M.F., 1997. Cellular solids: structures and properties. 2nd ed. Cambridge: Cambridge University Press.

Hodge A.M., Biener, J., Hayes J.R., Bythrow P.M., Volkert C.A., Hamza A.V., 2007. Scaling equation for yield strength of nanoporous open-cell foams. Acta Mater. 55, $1343-1349$. 
Huan T.N., Ganesh T., Kim K.S., Kim S., Han S.-H., Chung H., 2011. A three-dimensional gold nanodendrite network porous structure and its application for an electrochemical sensing. Biosens. Bioelectron. 27, $183-186$.

Hughes G.D., Smith S.D., Pande C.S. Johnson H.R., Armstrong R.W., 1986. Hall-petch strengthening for the microhardness of twelve nanometer grain diameter electrodeposited nickel. Scripta Metall. 20, $93-97$.

Lawn B.R., Howes V.R., 1981. Elastic recovery at hardness indentations. J. Mater. Sci., 16, $2745-2752$.

Linul E., Marsavina L., Voiconi T., Sadowski T., 2013. Study of factors influencing the mechanical properties of polyurethane foams under dynamic compression. J. Phys. Conf. Ser. 451, 012002.

Liu J., Cao L., Huang W., Li Z., 2011. Preparation of AuPt Alloy Foam Films and Their Superior Electrocatalytic Activity for the Oxidation of Formic Acid. ACS Appl. Mater. Interfaces 3, 3552 $-3558$.

Malgras V., Ataee-Esfahani H., Wang H., Jiang B., Li C., Wu K.C.-W., Ho Kim J., Yamauchi Y., 2015. Nanoarchitectures for Mesoporous Metals. Adv. Mater. 2015 (in press). DOI: 10.1002/adma.201502593

Mata M., Anglada M., Alcalá J., 2002. Contact deformation regimes around sharp indentations and the concept of the characteristic strain J. Mater. Res. 17, $964-976$.

Meza J.M., Abbes F., Troyon M., 2008. Penetration depth and tip radius dependence on the correction factor in nanoindentation measurements J. Mater. Res 23, $725-731$.

Oliver W.C., Pharr G.M., 1992. An imporved technique for determining hardness and elasticmodulus using load and displacement sensing indentation experiments. J. Mater. Res. 7, 1564 $-1580$.

Otsubo K., Haraguchi T., Sakata O., Fujiwara A., Kitagawa H., 2012. Step-by-step fabrication of a highly oriented crystalline three-dimensional pillared-layer-type metal-organic framework thin film confirmed by synchrotron x-ray diffraction. J. Am. Chem. Soc. 134, $9605-9608$. 
Pellicer E., Cabo M., Rossinyol E., Solsona P., Suriñach S., Baró M.D., Sort J., 2013. Nanocasting of Mesoporous In-TM ( $\mathrm{TM}=\mathrm{Co}, \mathrm{Fe}, \mathrm{Mn})$ Oxides: Towards 3D Diluted-Oxide Magnetic Semiconductor Architectures. Adv. Funct. Mater. 23, 900 - 911.

Pellicer E., Pané S., Panagiotopoulou V., Fusco S., Sivaraman K.M., Suriñach S., Baró M.D., Nelson B.J., Sort J., 2012. Localized electrochemical deposition of porous Cu-Ni microcolumns: insights into the growth mechanisms and the mechanical performance. Int. J. Electrochem. Sci. $7,4014-4029$.

Ramakrishnan N., Arunachalam V.S., 1993. Effective elastic-moduli of porous ceramic materials. J. Am. Ceram. Soc. 76, $2745-2752$.

Scaglione F., Rizzi P. , Battezzati L., 2012. De-alloying kinetics of an Au-based amorphous alloys. J. Alloys Compd. 536, S60 - S64.

Schwaiger R., Moser B., Dao M., Chollacoop N., Suresh S., 2003. Some critical experiments on the strain-rate sensitivity of nanocrystalline nickel. Acta Mater. 51, $5159-5172$.

Sun W., Kherani N.P., Hirschman K.D., Gadeken L.L., Fauchet P.M., 2005. A three-dimensional porous silicon p-n diode for betavoltaics and photovoltaics. Adv. Mater. 17, 1230 - 1233.

Thompson A.W., 1975. Yielding in nickel as a function of grain or cell size. Acta Metall. 23, $1337-1342$.

Williford R.E., Li X.S., Addleman R.S., Fryxell G.E., Baskaran S., Birnbaum J.C., Coyle C., Zemanian T.S., Wang C., Courtney A.R., 2005. Mechanical stability of templated mesoporous silica thin films. Microporous and Mesoporous Mater. 85, 260 - 266.

Xiong Q., Tu J., Lu Y., Chen J., Yu Y., Wang X., Gu C., 2012. Three-dimensional porous nano-Ni/ $\mathrm{Fe}_{3} \mathrm{O}_{4}$ composite film: enhanced electrochemical performance for lithium-ion batteries. J. Mater. Chem. 22, 18639 - 18645.

Xu Z.H., Li X.D., 2008. Effects of indenter geometry and material properties on the correction factor of Sneddon's relationship for nanoindentation of elastic and elastic-plastic materials Acta Mater. 56, 1399 - 1405.

Zeeshan M.A., Esqué-de los Ojos D., Castro-Hartman P., Guerrero M., Nogués J., Suriñach S., Baró M.D., Nelson B.J., Pané S., Pellicer E., Sort J., 2015. Electrochemically synthesized 
amorphous and crystalline nanowires: dissimilar nano-mechanical behavior in comparison with homologous flat films. Nanoscale DOI: 10.1039/c5nr04398k 